\title{
Flux compactification for the critical non-Abelian vortex and quark masses
}

\author{
A. Yung $(0$ \\ National Research Center “Kurchatov Institute,” Petersburg Nuclear Physics Institute, \\ Gatchina, St. Petersburg 188300, Russia \\ and William I. Fine Theoretical Physics Institute, University of Minnesota, \\ Minneapolis, Minnesota 55455, USA
}

(Received 17 May 2021; accepted 15 June 2021; published 6 July 2021)

\begin{abstract}
It has been shown that non-Abelian solitonic vortex strings supported in four-dimensional (4D) $\mathcal{N}=2$ supersymmetric QCD (SQCD) with the $\mathrm{U}(N=2)$ gauge group and $N_{f}=4$ quark flavors behave as critical superstrings. In addition to four translational moduli, the non-Abelian strings under consideration carry six orientational and size moduli. Together they form a ten-dimensional space required for a superstring to be critical. The target space of the string sigma model is a product of the flat four-dimensional space $\mathbb{R}^{4}$ and a Calabi-Yau noncompact threefold $Y_{6}$, namely, the conifold. The spectrum of low lying closed string states in the associated type IIA string theory was found and interpreted as a spectrum of hadrons in $4 \mathrm{D} \mathcal{N}=2 \mathrm{SQCD}$. In particular, the lowest string state appears to be a massless Bogomolny-Prasad-Sommerfeld baryon associated with the deformation of the complex structure modulus $b$ of the conifold. Here we address a problem of switching on quark masses in 4D SQCD, which classically breaks the world sheet conformal invariance in the string sigma model. To avoid this problem we follow a standard string theory approach and use a flux "compactification" to lift the complex structure modulus of the conifold. Namely, we find a solution of supergravity equations of motion with nonzero Neveu-Schwarz 3-form flux. It produces a potential for the baryon $b$, which leads to the runaway vacuum. Using field theory arguments, we interpret 3-form flux in terms of a particular choice of quark masses in 4D SQCD. At the runaway vacuum the conifold degenerates to lower dimensions. We interpret this as a flow from a non-Abelian string to an Abelian one.
\end{abstract}

DOI: 10.1103/PhysRevD.104.025007

\section{INTRODUCTION}

It was shown in [1] that the non-Abelian solitonic vortex string in four-dimensional (4D) $\mathcal{N}=2$ supersymmetric QCD (SQCD) with the $\mathrm{U}(\mathrm{N}=2)$ gauge group and $N_{f}=4$ flavors of quark hypermultiplets becomes a critical superstring. Non-Abelian vortices were first found in $\mathcal{N}=2$ $\mathrm{SQCD}$ with the gauge group $\mathrm{U}(N)$ and $N_{f} \geq N$ flavors of quarks [2-5]. The non-Abelian vortex string is $1 / 2$ Bogomolny-Prasad-Sommerfeld (BPS) saturated and, therefore, has $\mathcal{N}=(2,2)$ supersymmetry on its world sheet. In addition to four translational moduli characteristics of the Abrikosov-Nielsen-Olesen strings [6], the nonAbelian string carries orientational moduli, as well as the size moduli if $N_{f}>N$ [2-5] (see [7-10] for reviews). Their dynamics are described by the effective twodimensional (2D) sigma model on the string world sheet with the target space

Published by the American Physical Society under the terms of the Creative Commons Attribution 4.0 International license. Further distribution of this work must maintain attribution to the author(s) and the published article's title, journal citation, and DOI. Funded by SCOAP .

$$
\mathcal{O}(-1)_{\mathbb{C P}}^{\oplus\left(N_{f}-N\right)}
$$

which we will refer to as the weighted $C P$ model $\left[\mathrm{WCP}\left(N, N_{f}-N\right)\right]$.

For $N_{f}=2 N$ the world sheet sigma model becomes conformal. Moreover, for $N=2$ the number of the orientational/size moduli is six and they can be combined with four translational moduli to form a ten-dimensional (10D) space required for a superstring to become critical $[1,11]$. In this case, the target space of the world sheet 2D theory on the non-Abelian vortex string is $\mathbb{R}^{4} \times Y_{6}$, where $Y_{6}$ is a noncompact six-dimensional Calabi-Yau (CY) manifold, the conifold $[12,13]$.

Non-Abelian vortices in $\mathrm{U}(N)$ theories are topologically stable and cannot be broken. In particular, monopoles confined by the non-Abelian string are junctions of two strings with different orientations rather then string endpoints (see review [9]). Therefore the finite-length strings are closed.

Thus, we focus on the closed strings and consider CY "compactification" (Kaluza-Klein reduction) of 10D string theory associated with the non-Abelian vortex to $4 \mathrm{D}$ on the 
conifold $Y_{6}{ }^{1}$ The goal is to identify the closed string states with the hadrons of $4 \mathrm{D} \mathcal{N}=2 \mathrm{SQCD}$. The vortex string theory at hand was identified as the type IIA string theory [11].

The first step of this program, namely identifying massless string states, was carried out in $[11,14]$ using supergravity approximation. It turns out that most of the massless modes have non-normalizable wave functions over the noncompact conifold $Y_{6}$, i.e., they are not localized in $4 \mathrm{D}$ and, hence, cannot be interpreted as dynamical states in 4D SQCD. In particular, the 4D graviton and unwanted vector multiplet associated with deformations of the Kähler form of the conifold are absent. However, a single massless BPS hypermultiplet was found at the self-dual point at strong coupling. It is associated with deformations of a complex structure of the conifold and was interpreted as a composite $4 \mathrm{D}$ baryon, $b{ }^{2}$

Later low lying massive non-BPS 4D states were found in $[15,16]$ using the little string theory approach (see [17] for a review).

In this paper we consider $4 \mathrm{D} \mathcal{N}=2 \mathrm{SQCD}$ with nonzero quark masses. Quark masses are the only deformations of the 4D SQCD with given gauge and flavor groups allowed by $\mathcal{N}=2$ supersymmetry, which do not involve higher-derivative operators. It is interesting to study the string theory response to these deformations. However, making quarks massive lifts some of the orientational and size moduli of the non-Abelian string. Generically, this breaks the conformal invariance of the world sheet $\mathbb{W C P}(2,2)$ model (at least classically) preventing the formulation of the string theory in this case. This problem is puzzling because from the standpoint of 4D SQCD switching on quark masses is physically the most natural deformation one can consider.

To overcome this difficulty, instead of attempting to interpret mass terms for orientational/size moduli in the $\mathbb{W C P}(2,2)$ model in terms of 10D supergravity, we propose from the very beginning to use a flux "compactification" to describe 4D quark masses in the string theory framework. The motivation is that fluxes generically induce a potential for CY moduli lifting flat directions (see, for example, [18] for a review). On the other hand, in 4D theory we expect that quark masses also induce a potential for the massless baryon $b$ associated with the conifold complex structure modulus. In particular, we know that it acquires the mass

$$
m_{b}=\left|m_{1}+m_{2}-m_{3}-m_{4}\right|
$$

at nonzero $m_{A}$ dictated for a BPS state by its baryonic charge [19], where $m_{A}, A=1, \ldots, 4$ are quark masses. Thus, we expect that fluxes in 10D supergravity can be interpreted in terms of quark masses of 4D SQCD.

\footnotetext{
${ }^{1}$ Here and below we use the term "compactification" in quotation marks because the conifold is a noncompact CY space.

${ }^{2}$ The definition of the baryonic charge is nonstandard and will be given below in Sec. II.
}

Generically, fluxes modify the metric so that the deformed background is a solution of 10D supergravity equations of motion. This guarantees the conformal invariance of the deformed world sheet sigma model.

It is known that for type IIA CY "compactifications" the potential for the Kähler form moduli arise from RamondRamond (RR) even-form fluxes, while the potential for complex structure moduli is induced by the Neveu-Schwarz (NS) 3-form flux $H_{3}[20,21]$. Since we are interested in the potential for the conifold complex structure modulus $b$, we consider the NS 3 -form $H_{3}$. It does not break $\mathcal{N}=2$ supersymmetry in 4D theory [20].

We use a perturbation theory to solve 10D gravity equations, solving first equations of motion for $\mathrm{H}_{3}$ using the conifold metric. The back reaction on the metric and the dilaton arise in the quadratic order in $H_{3}$ and we neglect this effect to the leading order at small $H_{3}$.

Note that if we keep the vacuum expectation value (VEV) of the baryon $b$ large the curvature of the conifold is everywhere small and the gravity approximation is justified.

It turns out that $\mathrm{H}_{3}$ flux does not generate a mass term (1.2) for the baryon $b$. The mass term vanishes due to the noncompactness of the conifold. Instead, the $\mathrm{H}_{3}$ flux produces a potential leading to a runaway vacuum for $b$. We still look for the interpretation of the $\mathrm{H}_{3}$ flux in terms of quark masses. The reason for this is that there is no other deformation in 4D SQCD (preserving $\mathcal{N}=2$ supersymmetry) which produces a scalar potential, except mass deformation. Using field theory arguments we relate $\mathrm{H}_{3}$ to a particular choice of quark masses in 4D SQCD. At the runaway vacuum the deformed conifold degenerates to lower dimensions. We interpret this as a flow from a nonAbelian string to an Abelian one.

The paper is organized as follows. In Sec. II we briefly review $4 \mathrm{D} \mathcal{N}=2 \mathrm{SQCD}$ and the world sheet sigma model on the non-Abelian string. Next, we review the massless baryon $b$ as a deformation of the complex structure of the conifold and present the conifold metric. In Sec. III we solve equations of motion for the 3 -form $\mathrm{H}_{3}$ and show that $\mathrm{H}_{3}$ does not induce a mass (1.2) for the $b$-baryon. Also, we derive the potential for $b$ leading to the runaway vacuum. To better understand the behavior of the potential at large $b$ in Sec. IV, we solve the equations of motion for $\mathrm{H}_{3}$ using the deformed conifold metric and calculate the potential at large $b$. In Sec. V we interpret the $H_{3}$-form in terms of quark masses in $4 \mathrm{D}$ SQCD. We also discuss the interpretation of the degeneration of the conifold at the runaway vacuum as a flow from a non-Abelian string to an Abelian one. Section VI summarizes our conclusions.

\section{NON-ABELIAN CRITICAL VORTEX STRING}

\section{A. Four-dimensional $\mathcal{N}=2$ SQCD}

As was already mentioned, non-Abelian vortex strings were first found in $4 \mathrm{D} \mathcal{N}=2 \mathrm{SQCD}$ with the gauge group 
$\mathrm{U}(N)$ and $N_{f} \geq N$ quark flavors supplemented by the Fayet-Iliopoulos (FI) [22] term with parameter $\xi$ [2-5]. See, for example, [9] for a detailed review of this theory. Here, we just mention that at weak coupling $g^{2} \ll 1$, this theory is in the Higgs phase in which the scalar components of the quark multiplets (squarks) develop VEVs. These VEVs break the $\mathrm{U}(N)$ gauge group, Higgsing all gauge bosons. The Higgsed gauge bosons combine with the screened quarks to form long $\mathcal{N}=2$ multiplets with mass $m_{G} \sim g \sqrt{\xi}$.

The global flavor $\mathrm{SU}\left(N_{f}\right)$ is broken down to the socalled color-flavor locked group. The resulting global symmetry is

$$
\mathrm{SU}(N)_{C+F} \times \mathrm{SU}\left(N_{f}-N\right) \times \mathrm{U}(1)_{B}
$$

(see [9] for more details).

The unbroken global $\mathrm{U}(1)_{B}$ factor above is identified with a baryonic symmetry. Note that what is usually identified as the baryonic $\mathrm{U}(1)$ charge is a part of our $4 \mathrm{D}$ theory gauge group. "Our" $\mathrm{U}(1)_{B}$ is an unbroken by the squark VEVs combination of two U(1) symmetries: the first is a subgroup of the flavor $\mathrm{SU}\left(N_{f}\right)$, and the second is the global $\mathrm{U}(1)$ subgroup of $\mathrm{U}(N)$ gauge symmetry.

As was already noted, we consider $\mathcal{N}=2 \mathrm{SQCD}$ in the Higgs phase. $N$ squarks condense; therefore, non-Abelian vortex strings confine monopoles. In the $\mathcal{N}=24 \mathrm{D}$ theory, these strings are $1 / 2$ BPS-saturated; hence, their tension is determined exactly by the FI parameter,

$$
T=2 \pi \xi
$$

However, as we already mentioned, the monopoles cannot be attached to the string end points. In fact, in the $\mathrm{U}(N)$ theories confined monopoles are junctions of two distinct elementary non-Abelian strings [4,5,23] (see [9] for a review). As a result, in four-dimensional $\mathcal{N}=2 \mathrm{SQCD}$ we have monopole-anti-monopole mesons in which the monopole and antimonopole are connected by two confining strings. In addition, in the $\mathrm{U}(N)$ gauge theory we can have baryons appearing as closed "necklace" configurations of $N \times$ (integer) monopoles [9]. For the U(2) gauge group the important example of a baryon consists of four monopoles [19].

Below we focus on the particular case $N=2$ and $N_{f}=4$ because, as was mentioned in the Introduction, in this case $4 \mathrm{D} \mathcal{N}=2 \mathrm{SQCD}$ supports non-Abelian vortex strings which behave as critical superstrings [1]. Also, for $N_{f}=2 N$ the gauge coupling $g^{2}$ of the 4D SQCD does not run; the $\beta$ function vanishes. However, the conformal invariance of the 4D theory is explicitly broken by the FI parameter $\xi$, which defines VEV's of quarks. The FI parameter is not renormalized.

Both stringy monopole-anti-monopole mesons and monopole baryons with spins $J \sim 1$ have masses determined by the string tension $\sim \sqrt{\xi}$, and are heavier at weak coupling $g^{2} \ll 1$ than perturbative states with masses $m_{G} \sim g \sqrt{\xi}$. Thus, they can decay into perturbative states ${ }^{3}$ and, in fact, at weak coupling we do not expect them to appear as stable states.

Only in the strong coupling domain $g^{2} \sim 1$ do we expect (at least some of) the stringy mesons and baryons to become stable. These expectations were confirmed in $[11,15]$ where low lying string states in the string theory for the critical non-Abelian vortex were found at the selfdual point at strong coupling.

Below we introduce small quark masses $m_{A}, A=1, \ldots 4$, assuming that the two first squark flavors with masses $m_{1}$ and $m_{2}$ develop VEVs.

\section{B. World sheet sigma model}

The presence of the color-flavor locked group $\mathrm{SU}(N)_{C+F}$ is the reason for the formation of nonAbelian vortex strings [2-5]. The most important feature of these vortices is the presence of the orientational zero modes. As was already mentioned, in $\mathcal{N}=2$ SQCD these strings are $1 / 2$ BPS saturated.

Let us briefly review the model emerging on the world sheet of the non-Abelian string [9].

The translational moduli fields are described by the Nambu-Goto action and decouple from all other moduli. Below we focus on internal moduli.

If $N_{f}=N$, the dynamics of the orientational zero modes of the non-Abelian vortex, which become orientational moduli fields on the world sheet, are described by the 2D $\mathcal{N}=(2,2)$ supersymmetric $\mathbb{C P}(N-1)$ model.

If one adds additional quark flavors, non-Abelian vortices become semilocal-they acquire size moduli [24]. In particular, for the non-Abelian semilocal vortex in $\mathrm{U}(2) \mathcal{N}=2$ SQCD with four flavors, in addition to the complex orientational moduli $n^{P}$ (here $P=1,2$ ), we must add two complex size moduli $\rho^{K}$ (where $K=3,4$ ) (see [2,5,24-27]).

The effective theory on the string world sheet is a two-dimensional $\mathcal{N}=(2,2)$ supersymmetric $\mathbb{W} \mathbb{C P}(2,2)$ model $[1,11,14]{ }^{4}$

This model describes the internal dynamics of the nonAbelian semilocal string. For details, see, e.g., the review [9].

\footnotetext{
${ }^{3}$ Their quantum numbers, with respect to the global group (2.1), allow these decays (see [9]).

${ }^{4}$ Both the orientational and the size moduli have logarithmically divergent norms (see, e.g., [25]). After an appropriate infrared regularization, logarithmically divergent norms can be absorbed into the definition of relevant two-dimensional fields [25]. In fact, the world sheet theory on the semilocal non-Abelian string is not exactly the $\mathbb{W} C \mathbb{P}(N, \tilde{N})$ model [27]—-there are minor differences. The actual theory is called the $z n$ model. Nevertheless, it has the same infrared physics as the model (2.3) [28] (see also [29]).
} 
The $\mathbb{W C P}(2,2)$ sigma model can be defined as a low energy limit of the U(1) gauge theory [30], which corresponds to taking the limit of infinite gauge coupling, $e^{2} \rightarrow \infty$, in the action below. The bosonic part of the action reads ${ }^{5}$

$$
\begin{aligned}
S= & \int d^{2} x\left\{\left|\nabla_{\alpha} n^{P}\right|^{2}+\left|\tilde{\nabla}_{\alpha} \rho^{K}\right|^{2}+\frac{1}{4 e^{2}} F_{\alpha \beta}^{2}+\frac{1}{e^{2}}\left|\partial_{\alpha} \sigma\right|^{2}\right. \\
& +2\left|\sigma+\frac{m_{P}}{\sqrt{2}}\right|^{2}\left|n^{P}\right|^{2}+2\left|\sigma+\frac{m_{K}}{\sqrt{2}}\right|^{2}\left|\rho^{K}\right|^{2} \\
& \left.+\frac{e^{2}}{2}\left(\left|n^{P}\right|^{2}-\left|\rho^{K}\right|^{2}-\operatorname{Re} \beta\right)^{2}\right\}, \\
P= & 1,2, \quad K=3,4 .
\end{aligned}
$$

Here, $m_{A}(A=1, \ldots, 4)$ are the so-called twisted masses (they coincide with $4 \mathrm{D}$ quark masses), while $\operatorname{Re} \beta$ is the inverse coupling constant (2D FI term). More exactly it is the real part of the complexified coupling constant introduced in Eq. (2.7) below.

The fields $n^{P}$ and $\rho^{K}$ have charges +1 and -1 with respect to the auxiliary $\mathrm{U}(1)$ gauge field, and the corresponding covariant derivatives in (2.3) are defined as

$$
\nabla_{\alpha}=\partial_{\alpha}-i A_{\alpha}, \quad \tilde{\nabla}_{\alpha}=\partial_{\alpha}+i A_{\alpha}
$$

respectively. The complex scalar field $\sigma$ is a superpartner of the U(1) gauge field $A_{\alpha}$.

Apart from the U(1) gauge symmetry, the sigma model (2.3) in the massless limit has a global symmetry group

$$
\mathrm{SU}(2) \times \mathrm{SU}(2) \times \mathrm{U}(1)_{B}
$$

i.e., exactly the same as the unbroken global group in the 4D theory at $N=2$ and $N_{f}=4$. The fields $n$ and $\rho$ transform in the following representations:

$$
n:(\mathbf{2}, \mathbf{1}, 0), \quad \rho:(\mathbf{1}, \mathbf{2}, 1) .
$$

Here the global "baryonic" $\mathrm{U}(1)_{B}$ symmetry is a classically unbroken (at $\beta>0$ ) combination of the global $\mathrm{U}(1)$ group, which rotates $n$ and $\rho$ fields with the same phases, plus $U(1)$ gauge symmetry, which rotates them with the opposite phases (see [11] for details). The nonzero twisted masses $m_{A}$ break each of the SU(2) factors in (2.5) down to $\mathrm{U}(1)$.

The $2 \mathrm{D}$ coupling constant $\operatorname{Re} \beta$ can be naturally complexified if we include the $\theta$ term in the action,

\footnotetext{
${ }^{5}$ Equation (2.3) and similar expressions below are given in Euclidean notation.
}

$$
\beta=\operatorname{Re} \beta+i \frac{\theta_{2 D}}{2 \pi},
$$

where $\theta_{2 D}$ is the $2 \mathrm{D} \theta$ angle.

The number of real bosonic degrees of freedom in the model (2.3) is $8-1-1=6$. Here, 8 is the number of real degrees of freedom of $n^{P}$ and $\rho^{K}$ fields and we subtracted one real constraint imposed by the $D$ term condition in (2.3),

$$
\left|n^{P}\right|^{2}-\left|\rho^{K}\right|^{2}=\operatorname{Re} \beta,
$$

in the limit $e^{2} \rightarrow \infty$ and one gauge phase eaten by the Higgs mechanism. As we already mentioned, these six internal degrees of freedom in the massless limit can be combined with four translational moduli to form a 10D space needed for a superstring to be critical.

At the quantum level, the coupling $\beta$ does not run in this theory. Thus, the $\mathbb{W} C \mathbb{P}(2,2)$ model is superconformal at zero masses $m_{A}=0$. Therefore, its target space is Ricci-flat and [being Kähler due to $\mathcal{N}=(2,2)$ supersymmetry] represents a noncompact Calabi-Yau manifold, namely the conifold $Y_{6}$ (see [13] for a review).

The $\mathbb{W C P}(2,2)$ model (2.3) with $m_{A}=0$ was used in $[1,11]$ to define the critical string theory for the nonAbelian vortex at hand.

Typically solitonic strings are "thick" and the effective world sheet theory, like the one in (2.3), has a series of unknown high derivative corrections in powers of $\partial / m_{G}$. The string transverse size is given by $1 / m_{G}$, where $m_{G} \sim$ $g \sqrt{\xi}$ is a mass scale of the gauge bosons and quarks forming the string. The string cannot be thin in a weakly coupled 4D SQCD because at weak coupling $m_{G} \sim g \sqrt{T}$ and $m_{G}^{2}$ is always small in the units of the string tension $T$ [see (2.2)].

A conjecture was put forward in [1] that at strong coupling in the vicinity of a critical value $g_{c}^{2} \sim 1$ the non-Abelian string in the theory at hand becomes thin, and higher-derivative corrections in the world sheet theory (2.3) are absent. This is possible because the low energy sigma model (2.3) already describes a critical string and higher-derivative corrections are not required to improve its ultraviolet behavior (see [31] for the discussion of this problem). The above conjecture implies that $m_{G}\left(g^{2}\right) \rightarrow \infty$ at $g^{2} \rightarrow g_{c}^{2}$. As expected, the thin string produces linear Regge trajectories even for small spins [16].

It was also conjectured in [11] that $g_{c}$ corresponds to the value of the $2 \mathrm{D}$ coupling constant $\beta=0$. The motivation for this conjecture is that this value is a self-dual point for the $\mathbb{W C P}(2,2)$ model (2.3). Also, $\beta=0$ is a natural choice because at this point we have a regime change in the $\mathbb{W} C P(2,2)$ model. The resolved conifold defined by the $D$ term condition (2.8) develops a conical singularity at this point. The point $\beta=0$ corresponds to $\tau_{S W}=1$ in the $4 \mathrm{D}$ 
SQCD, where $\tau_{S W}$ is the complexified inverse coupling $\tau_{S W}=i \frac{8 \pi}{g^{2}}+\frac{\theta_{4 D}}{\pi}$, where $\theta_{4 D}$ is the 4D $\theta$ angle [19].

A version of the string-gauge duality for 4D SQCD was proposed [1]: at weak coupling this theory is in the Higgs phase and can be described in terms of quarks and Higgsed gauge bosons, while at strong coupling, hadrons of this theory can be understood as string states formed by the non-Abelian vortex string.

At nonzero quark masses $m_{A} \neq 0$, the model (2.3) is a mass deformation of the superconformal CY theory on the conifold. Generically, quark masses break the world sheet conformal invariance. The $\mathbb{W} \mathbb{C P}(2,2)$ model in $(2.3)$ can no longer be used to define a string theory for the nonAbelian vortex in the massive 4D SQCD.

\section{Massless 4D baryon}

In this section we briefly review the only $4 \mathrm{D}$ massless state found in the string theory of the critical non-Abelian vortex in the massless limit [11]. It is associated with the deformation of the conifold complex structure. As was already mentioned, all other massless string modes have non-normalizable wave functions over the conifold. In particular, the 4D graviton associated with a constant wave function over the conifold $Y_{6}$ is absent [11]. This result matches our expectations since we started with $\mathcal{N}=2$ SQCD in the flat four-dimensional space without gravity.

We can construct the U(1) gauge-invariant "mesonic" variables

$$
w^{P K}=n^{P} \rho^{K} .
$$

These variables are subject to the constraint

$$
\operatorname{det} w^{P K}=0 .
$$

Equation (2.10) defines the conifold $Y_{6}$. It has the Ricciflat Kähler metric and represents a noncompact Calabi-Yau manifold $[12,13,30]$. It is a cone which can be parametrized by the noncompact radial coordinate,

$$
\tilde{r}^{2}=\operatorname{Tr} \bar{w} w
$$

and five angles (see [12]). Its section at fixed $\tilde{r}$ is $S_{2} \times S_{3}$.

At $\beta=0$ the conifold develops a conical singularity, so both spheres $S_{2}$ and $S_{3}$ can shrink to zero. The conifold singularity can be smoothed out in two distinct ways: by deforming the Kähler form or by deforming the complex structure. The first option is called the resolved conifold and amounts to keeping a nonzero value of $\beta$ in (2.8). This resolution preserves the Kähler structure and Ricciflatness of the metric. If we put $\rho^{K}=0$ in (2.3) we get the $\mathbb{C P}(1)$ model with the sphere $S_{2}$ as a target space (with the radius $\sqrt{\beta}$ ). The resolved conifold has no normalizable zero modes. In particular, the modulus $\beta$ which becomes a scalar field in four dimensions has a non-normalizable wave function over the $Y_{6}$ and therefore is not dynamical [11].

If $\beta=0$ another option exists, namely a deformation of the complex structure [13]. It preserves the Kähler structure and the Ricci-flatness of the conifold and is usually referred to as the deformed conifold. It is defined by the deformation of Eq. (2.10), namely

$$
\operatorname{det} w^{P K}=b
$$

where $b$ is a complex parameter. Now the sphere $S_{3}$ can not shrink to zero-its minimal size is determined by $b$.

The modulus $b$ becomes a 4D complex scalar field. The effective action for this field was calculated in [11] using the explicit metric on the deformed conifold [12,32,33],

$$
S_{\text {kin }}(b)=T \int d^{4} x\left|\partial_{\mu} b\right|^{2} \log \frac{\tilde{R}_{\mathrm{IR}}^{2}}{|b|},
$$

where $\tilde{R}_{\mathrm{IR}}$ is the maximal value of the radial coordinate $\tilde{r}$ introduced as an infrared regularization of the logarithmically divergent $b$-field norm. Here the logarithmic integral at small $\tilde{r}$ is cut off by the minimal size of $S_{3}$, which is equal to $|b|$.

We see that the norm of the modulus $b$ turns out to be logarithmically divergent in the infrared. The modes with the logarithmically divergent norm are at the borderline between normalizable and non-normalizable modes. Usually such states are considered to be "localized" ones. We follow this rule. This scalar mode is localized near the conifold singularity in the same sense as the orientational and size zero modes are localized on the vortex string solution.

The field $b$ being massless can develop a VEV. Thus, we have a new Higgs branch in $4 \mathrm{D} \mathcal{N}=2$ SQCD which is developed only for the critical value of the $4 \mathrm{D}$ coupling constant $\tau_{S W}=1$ associated with $\beta=0$.

In [11] the massless state $b$ was interpreted as a baryon of $4 \mathrm{D} \mathcal{N}=2$ QCD. Let us explain this. From Eq. (2.12) we see that the complex parameter $b$ (which is promoted to a 4D scalar field) is a singlet with respect to both $\mathrm{SU}(2)$ factors in (2.5), i.e., the global world sheet group. ${ }^{6}$ What about its baryonic charge? From (2.6) and (2.12) we see that the $b$ state transforms as

$$
(\mathbf{1}, \mathbf{1}, 2) \text {. }
$$

In particular, it has the baryon charge $Q_{B}(b)=2$.

In type IIA superstring compactifications the complex scalar associated with deformations of the complex structure of the Calabi-Yau space enters as a $4 \mathrm{D} \mathcal{N}=2$ BPS hypermultiplet (see [18] for a review). Other components of

\footnotetext{
${ }^{6}$ Which is isomorphic to the $4 \mathrm{D}$ global group (2.1) for $N=2$, $N_{f}=4$.
} 
this hypermultiplet can be restored by $\mathcal{N}=2$ supersymmetry. In particular, the $4 \mathrm{D} \mathcal{N}=2$ hypermultiplet should contain another complex scalar $\tilde{b}$ with baryon charge $Q_{B}(\tilde{b})=-2$. In the stringy description this scalar comes from a ten-dimensional 3-form (see [18] for a review, as well as Sec. III C and the Appendix).

To conclude this section, let us present the explicit metric of the singular conifold (with both $\beta$ and $b$ equal to zero), which will be used in the next section. It has the form [12]

$d s_{6}^{2}=d r^{2}+\frac{r^{2}}{6}\left(e_{\theta_{1}}^{2}+e_{\varphi_{1}}^{2}+e_{\theta_{2}}^{2}+e_{\varphi_{2}}^{2}\right)+\frac{r^{2}}{9} e_{\psi}^{2}$,

where

$$
\begin{aligned}
e_{\theta_{1}} & =d \theta_{1}, \quad e_{\varphi_{1}}=\sin \theta_{1} d \varphi_{1}, \\
e_{\theta_{2}} & =d \theta_{2}, \quad e_{\varphi_{2}}=\sin \theta_{2} d \varphi_{2}, \\
e_{\psi} & =d \psi+\cos \theta_{1} d \varphi_{1}+\cos \theta_{2} d \varphi_{2} .
\end{aligned}
$$

Here, $r$ is another radial coordinate on the cone while the angles above are defined at $0 \leq \theta_{1,2}<\pi, 0 \leq \varphi_{1,2}<2 \pi$, $0 \leq \psi<4 \pi$.

The volume integral associated with this metric is

$$
(\mathrm{Vol})_{Y_{6}}=\frac{1}{108} \int r^{5} d r d \psi d \theta_{1} \sin \theta_{1} d \varphi_{1} d \theta_{2} \sin \theta_{2} d \varphi_{2} .
$$

The radial coordinate $\tilde{r}$, defined in terms of matrix $w^{P K}$ [see (2.11)], is related to $r$ in (2.15) via [12]

$$
r^{2}=\frac{3}{2} \tilde{r}^{4 / 3}
$$

\section{NS 3-FORM}

Now we switch on small quark masses $m_{A}, A=1, \ldots, 4$. We will use the effective 10D supergravity approach to find the deformed background for our non-Abelian vortex string. As we already explained in the Introduction, instead of attempting to interpret twisted mass terms in (2.3) in terms of 10D gravity, we take a different route. We exploit the standard string theory approach of flux "compactifications" to 4D. Namely, we look for the solution of 10D gravity equations of motion with nonzero NS 3 -form, $\mathrm{H}_{3}$, and then interpret $\mathrm{H}_{3}$ in terms of quark masses. Note that the 3-form $\mathrm{H}_{3}$ flux does not break $\mathcal{N}=2$ supersymmetry in $4 \mathrm{D}[20]$.

The motivation to consider the NS 3 -form $\mathrm{H}_{3}$ is as follows. We expect that quark masses should lift the Higgs branch associated with the massless baryon $b$. In particular, the mass term (1.2) for the baryon $b$ appears for generic values of quark masses as dictated for a BPS state with a nonzero baryonic charge [19]. On the other hand, generically, in type IIA compactifications a potential for Kähler moduli is generated by even-form RR fluxes, while the potential for complex structure moduli (like our $b$-modulus) is induced by the NS 3-form flux [20].

As we already mentioned, for solving gravity equations of motion we will use the perturbation theory at small $\mathrm{H}_{3}$. Namely, we will solve equations of motion for $\mathrm{H}_{3}$ using the conifold metric, neglecting the back reaction on the metric and the dilaton. These effects appear in the quadratic order in $\mathrm{H}_{3}$. We also assume that the VEV of the baryon $b$ is large to make sure that the curvature of the conifold is everywhere small. This justifies the gravity approximation.

\section{A. Solution for NS 3-form at large $r$}

The bosonic part of the action of the type IIA supergravity in the Einstein frame is given by

$$
\begin{aligned}
S_{10 D}= & \int d^{10} x \sqrt{-G}\left\{R-\frac{1}{2} G^{M N} \partial_{M} \Phi \partial_{N} \Phi\right. \\
& \left.-\frac{e^{-\Phi}}{12} H_{M N L} H^{M N L}-\frac{1}{2} e^{\frac{\Phi}{2}} F_{4}^{2}\right\} \\
& +T^{2} \int \frac{1}{2} H_{3} \wedge C_{3} \wedge d C_{3},
\end{aligned}
$$

where $G_{M N}$ and $\Phi$ are 10D metric and dilaton, and the string coupling $g_{s}=e^{\Phi}$. We also keep only the NS 2-form $B_{2}$ with the field strength $H_{3}=d B_{2}$ and the RR 3-form $C_{3}$ with the field strength $F_{4}=d C_{3}$. We will need the RR 3-form $C_{3}$ below to introduce the complex scalar $\tilde{b}$, the bosonic superpartner of $b$ in the 4D baryonic hypermultiplet.

In this section we will find a solution for the NS 3-form $\mathrm{H}_{3}$ at large values of the radial coordinate $r$. Large means that $\tilde{r}$ is much larger than the minimal radius of the $S_{3}$ of the deformed conifold, which is equal to $\sqrt{|b|}$ [see (2.12)]. This translates into $r \gg|b|^{1 / 3}$ [see (2.18)]. At large $r$ we can neglect the deformation of the conifold and use the metric of the singular conifold. Thus, the 10D space has the structure $\mathbb{R}^{4} \times Y_{6}$ with the metric

$d s_{10}^{2}=T\left[-(d t)^{2}+\left(d x^{1}\right)^{2}+\left(d x^{2}\right)^{2}+\left(d x^{3}\right)^{2}\right]+d s_{6}^{2}$,

where the metric of $Y_{6}$ is given by (2.15) and $T$ is the string tension (2.2).

For 2-form $B_{2}$, we use the ansatz introduced in [33-36] for type IIB "compactifications" on the conifold. Namely, we write

$$
B_{2}=f_{1}(r) e_{\theta_{1}} \wedge e_{\varphi_{1}}+f_{2}(r) e_{\theta_{2}} \wedge e_{\varphi_{2}},
$$

where $f_{1}(r)$ and $f_{2}(r)$ are functions of the radial coordinate $r$, while angle differentials are defined in (2.16). This gives, for the 3 -form field strength, 


$$
H_{3}=f_{1}^{\prime} d r \wedge e_{\theta_{1}} \wedge e_{\varphi_{1}}+f_{2}^{\prime} d r \wedge e_{\theta_{2}} \wedge e_{\varphi_{2}},
$$

where prime denotes the derivative with respect to $r$. This 3-form is closed so the Bianchy identity is satisfied.

In order to find a nonzero solution for $\mathrm{H}_{3}$ we have to either introduce a source like a D-brane or impose a nontrivial boundary condition for $\mathrm{H}_{3}$. We use the latter option and specify the boundary condition for $\mathrm{H}_{3}$ at small $r$ in Sec. IV B.

The equation of motion for $H_{3}$ reads

$$
d\left(e^{-\Phi} * H_{3}\right)=e^{-\Phi} d\left(* H_{3}\right)=0,
$$

where $*$ denotes the Hodge star and, as we explained above, we neglect the back reaction on the dilaton to the leading order in small $\mathrm{H}_{3}$ and assume that the dilaton is constant.

The 10D-dual of $H_{3}$ is given by

$$
\begin{aligned}
* H_{3}= & \frac{T^{2}}{3} d x^{0} \wedge d x^{1} \wedge d x^{2} \wedge d x^{3} \wedge e_{\psi} \\
& \wedge\left(e_{\theta_{2}} \wedge e_{\varphi_{2}} r f_{1}^{\prime}+e_{\theta_{1}} \wedge e_{\varphi_{1}} r f_{2}^{\prime}\right) .
\end{aligned}
$$

Substituting this into the equation of motion (3.5) we find three equations:

$$
\partial_{r}\left(r f_{1}^{\prime}\right)=0, \quad \partial_{r}\left(r f_{2}^{\prime}\right)=0,
$$

and

$$
f_{1}^{\prime}+f_{2}^{\prime}=0
$$

The nonzero solution to these equations has the form

$$
f_{1}=-f_{2}=\mu_{1} \log r,
$$

where $\mu_{1}$ is a small real parameter which we will interpret in terms of quark masses in Sec. V.

This gives, for the 3-form $\mathrm{H}_{3}$,

$$
H_{3}=\mu_{1} \frac{d r}{r} \wedge\left(e_{\theta_{1}} \wedge e_{\varphi_{1}}-e_{\theta_{2}} \wedge e_{\varphi_{2}}\right) .
$$

Essentially, this coincides with the solution obtained in [33-35] for the NS 3-form in type IIB "compactifications" on the conifold.

\section{B. A generalization}

In this section we make a generalization of our solution (3.10) for $\mathrm{H}_{3}$. Let us define two real 3-forms on $Y_{6}$,

$$
\alpha_{3} \equiv \frac{d r}{r} \wedge\left(e_{\theta_{1}} \wedge e_{\varphi_{1}}-e_{\theta_{2}} \wedge e_{\varphi_{2}}\right)
$$

and

$$
\beta_{3} \equiv e_{\psi} \wedge\left(e_{\theta_{1}} \wedge e_{\varphi_{1}}-e_{\theta_{2}} \wedge e_{\varphi_{2}}\right) .
$$

They are both closed [34,35]:

$$
d \alpha_{3}=0, \quad d \beta_{3}=0 .
$$

Moreover, using the conifold metric (2.15) one can check that their 10D-duals are given by

$$
\begin{aligned}
& * \alpha_{3}=-\frac{T^{2}}{3} d x^{0} \wedge d x^{1} \wedge d x^{2} \wedge d x^{3} \wedge \beta_{3}, \\
& * \beta_{3}=3 T^{2} d x^{0} \wedge d x^{1} \wedge d x^{2} \wedge d x^{3} \wedge \alpha_{3},
\end{aligned}
$$

where the first relation was already shown in the previous section [see (3.6)].

The above relations ensure that both 10D-dual forms satisfy equations of motion

$$
d * \alpha_{3}=0, \quad d * \beta_{3}=0
$$

Therefore, we can generalize our solution (3.10) for $H_{3}$ by writing

$$
H_{3}=\mu_{1} \alpha_{3}+\frac{\mu_{2}}{3} \beta_{3},
$$

where $\mu_{2}$ is another real parameter, while the factor $\frac{1}{3}$ is introduced for convenience. This $\mathrm{H}_{3}$-form satisfies both the Bianchi identity and equations of motion (3.5).

3-Forms (3.11) and (3.12) form a basis similar to the simplectic basis of the harmonic $\alpha$ and the $\beta 3$-forms for compact CYs (see, for example, review [18]). In particular,

$$
\int_{Y_{6}} \alpha_{3} \wedge \alpha_{3}=\int_{Y_{6}} \beta_{3} \wedge \beta_{3}=0
$$

while

$$
\int_{Y_{6}} \alpha_{3} \wedge \beta_{3} \sim-\int \frac{d r}{r} \sim-\log \frac{R_{\mathrm{IR}}^{3}}{|b|} .
$$

Here $R_{\mathrm{IR}}$ is the maximal value of the radial coordinate $r$ introduced to regularize the infrared logarithmic divergence, while at small $r$ the integral is cut off by the minimal size of $S_{3}$ which is equal to $|b|$. This logarithmic behavior will play an important role below. Note that this logarithm is similar to the one which determines the metric for the b-baryon in (2.13). ${ }^{7}$

\section{Mass term for $\tilde{b}$-baryon}

Generically, in type IIA compactifications the potential for complex structure moduli comes from two sources: the topological term and the kinetic term for $B_{2}$ in the 10D

\footnotetext{
${ }^{7}$ Note that $R_{\mathrm{IR}}^{3} \sim \tilde{R}_{\mathrm{IR}}^{2}[$ see (2.18)].
} 
action, namely, the last and the first terms in the second line of (3.1), respectively [20]. In this section we consider the topological term.

Following the standard procedure for compactifications on CY 3-folds [18], we expand the 10D potential $C_{3}$ as

$$
C_{3}=C_{3}^{4 D}+\tilde{b}_{1} \alpha_{3}+\frac{1}{3} \tilde{b}_{2} \beta_{3} .
$$

Here $C_{3}^{4 D}(x)$ is the part of the $10 \mathrm{D}$ potential $C_{3}$ oriented in 4D which depends only on 4D coordinates $x_{\mu}$. Real 4D scalar fields $\tilde{b}_{1}$ and $\tilde{b}_{2}$ can be combined into a complex scalar, $\tilde{b}(x)=\tilde{b}_{1}(x)+i \tilde{b}_{2}(x)$, which, together with the complex scalar $b$, forms a bosonic part of the 4D baryonic hypermultiplet.

In the Appendix we calculate kinetic terms for 4D scalars $\tilde{b}_{1,2}$ using the last two last terms in (3.19) to calculate the 4-form field strength $F_{4}=d C_{3}$. We show that scalars $\tilde{b}_{1,2}$ acquire the same logarithmic metric as the complex scalar $b$ [see (2.13)], as dictated by $4 \mathrm{D} \mathcal{N}=2$ supersymmetry.

In order to obtain the mass term for $\tilde{b}$, we, following [20], saturate the last factor $d C_{3}$ in the topological term in (3.1) by the $4 \mathrm{D}$ potential, $C_{3}^{4 D}(x)$. Dualizing this to a $4 \mathrm{D}$ scalar $e(x), d C_{3}^{4 D}(x)_{\mu \nu \delta \gamma}=e(x) \varepsilon_{\mu \nu \delta \gamma}$, we get

$$
\int \frac{1}{2} H_{3} \wedge C_{3} \wedge d C_{3}=\frac{1}{2} \int d^{4} x\left\{e \int_{Y_{6}} H_{3} \wedge C_{3}\right\} .
$$

Now we substitute here our solution for the $H_{3}$-form (3.16) and the expansion (3.19). This gives the contribution to the 4D action,

$$
T^{2} \int d^{4} x e\left(\mu_{2} \tilde{b}_{1}-\mu_{1} \tilde{b}_{2}\right) \log \frac{R_{\mathrm{IR}}^{3}}{|b|},
$$

where we used (3.17) and (3.18).

Combining this with the kinetic term for $C_{3}^{4 D}$ in (3.1), we get the part of the 4D action depending on the scalar $e$ :

$$
\begin{aligned}
S_{4 D}^{e}= & T^{2} \int d^{4} x\left\{\frac{1}{2}(\mathrm{Vol})_{Y_{6}} e^{\Phi / 2} e^{2}\right. \\
& \left.+e\left(\mu_{2} \tilde{b}_{1}-\mu_{1} \tilde{b}_{2}\right) \log \frac{R_{\mathrm{IR}}^{3}}{|b|}\right\} .
\end{aligned}
$$

Here, $(\mathrm{Vol})_{Y_{6}} \sim R_{\mathrm{IR}}^{6}$ is the infinite volume of the conifold [see (2.17)]. Integrating the scalar $e$ out, we finally get the mass term for $\tilde{b}$ :

$$
\begin{aligned}
S_{\text {mass }}(\tilde{b})= & -\frac{T^{2} e^{\Phi / 2}}{2(\mathrm{Vol})_{Y_{6}}} \int d^{4} x\left(\mu_{2} \tilde{b}_{1}-\mu_{1} \tilde{b}_{2}\right)^{2} \\
& \times\left.\left(\log \frac{R_{\mathrm{IR}}^{3}}{|b|}\right)^{2}\right|_{R_{\mathrm{IR}} \rightarrow \infty} \rightarrow 0 .
\end{aligned}
$$

We see that the mass term for the baryon $\tilde{b}$ vanishes. In contrast to type IIA compactifications on compact CY spaces, where the topological term in (3.1) produces mass terms for scalar superpartners of complex structure moduli [20], in our case it goes to zero due to the non-normalizability of $C_{3}^{4 D}$. In terms of quark masses we interpret the result in (3.23) as follows. The NS 3-form $\mathrm{H}_{3}$ corresponds to a particular choice of quark masses for which $m_{b}=0$ [see (1.2)]. We will come back to the interpretation of $H_{3}$ in terms of quark masses later in Sec. V.

In fact, this result is a test of the consistency of our approach. To see this, note that the mass term (3.23) gives mass to a particular combination of $\tilde{b}_{1}$ and $\tilde{b}_{2}$, while the orthogonal combination remains massless. If this term were nonzero this would signal the breaking of $\mathcal{N}=2$ supersymmetry in 4D. On the other hand, we know that quark masses do not break $\mathcal{N}=2$ supersymmetry in 4D SQCD.

\section{The potential for the baryon $b$}

As we already mentioned in the type IIA compactifications, another source of the potential for complex structure moduli come from the kinetic term for $B_{2}$ in (3.1). Writing this term as

$$
-\frac{e^{-\Phi}}{12} \int H_{3} \wedge * H_{3}
$$

and substituting here our solution (3.16), we get

$$
-T^{2} e^{-\Phi}\left(\mu_{1}^{2}+\mu_{2}^{2}\right) \int d^{4} x \log \frac{R_{\mathrm{IR}}^{3}}{|b|},
$$

where we used (3.14) and (3.18). Thus, the scalar potential has the form

$$
V(b)=\text { const } T^{2} e^{-\Phi}\left(\mu_{1}^{2}+\mu_{2}^{2}\right) \log \frac{R_{\mathrm{IR}}^{3}}{|b|} .
$$

The potential (3.26) is proportional to the same infrared logarithm which enters the metric (2.13) for the baryon $b$. We see that the Higgs branch for $b$ is lifted and we have a runaway vacuum with VEV

$$
\langle|b|\rangle \rightarrow R_{\mathrm{IR}}^{3} \rightarrow \infty
$$

In fact, our solution (3.16) is found using the metric of the singular conifold and therefore is valid at $r \gg|b|^{1 / 3}$. Thus, the potential (3.26) can be trusted at small $|b|$ where the logarithm is large and we cannot really use it at $|b| \sim R_{\mathrm{IR}}^{3}$. In the next section, we consider the case of large $|b|$ and confirm our conclusion in (3.27) that the VEV of the baryon $b$ tends to infinity. 


\section{DEFORMED CONIFOLD}

In the previous section we have seen that the 3 -form $\mathrm{H}_{3}$ lifts the Higgs branch for the baryon $b$ and pushes the VEV of $b$ towards large $|b|,|b| \sim R_{\mathrm{IR}}^{3}$. To study the behavior of the potential $V(b)$ in this case we have to find the solution for $\mathrm{H}_{3}$ on the deformed conifold assuming that the radial coordinate $r \sim|b|^{1 / 3}$. First, we briefly review the metric of the deformed conifold and then we solve equations of motion for $\mathrm{H}_{3}$.

\section{A. Metric of the deformed conifold}

The metric of the deformed conifold has the form $[12,32,33]$

$$
\begin{aligned}
d s_{6}^{2}= & \frac{1}{2}|b|^{2 / 3} K(\tau)\left\{\frac{1}{3 K^{3}(\tau)}\left(d \tau^{2}+e_{\psi}^{2}\right)+\cosh ^{2} \frac{\tau}{2}\left(g_{3}^{2}+g_{4}^{2}\right)\right. \\
& \left.+\sinh ^{2} \frac{\tau}{2}\left(g_{1}^{2}+g_{2}^{2}\right)\right\},
\end{aligned}
$$

where angle differentials are defined as

$$
\begin{array}{rlrl}
g_{1} & =-\frac{1}{\sqrt{2}}\left(e_{\phi_{1}}+e_{3}\right), & g_{2} & =\frac{1}{\sqrt{2}}\left(e_{\theta_{1}}-e_{4}\right), \\
g_{3}=-\frac{1}{\sqrt{2}}\left(e_{\phi_{1}}-e_{3}\right), & g_{4}=\frac{1}{\sqrt{2}}\left(e_{\theta_{1}}+e_{4}\right),
\end{array}
$$

while

$$
\begin{aligned}
& e_{3}=\cos \psi \sin \theta_{2} d \varphi_{2}-\sin \psi d \theta_{2}, \\
& e_{4}=\sin \psi \sin \theta_{2} d \varphi_{2}+\cos \psi d \theta_{2}
\end{aligned}
$$

[see also (2.16)].

Here,

$$
K(\tau)=\frac{(\sinh 2 \tau-2 \tau)^{1 / 3}}{2^{1 / 3} \sinh \tau}
$$

and the new radial coordinate, $\tau$, is defined as

$$
\tilde{r}^{2}=|b| \cosh \tau=\left(\frac{2}{3}\right)^{\frac{3}{2}} r^{3}
$$

In the limit of large $\tau$, the metric (4.1) reduces to the metric (2.15) of the singular conifold.

Results of the previous section show that we have a runaway vacuum with $|b| \sim R_{\mathrm{IR}}^{3}$, so we are interested in the metric (4.1) in the limit of small $\tau, \tau \ll 1$. In this limit, the metric of the deformed conifold takes the form

$$
\begin{aligned}
\left.d s_{6}^{2}\right|_{\tau \rightarrow 0}= & \frac{1}{2}|b|^{2 / 3}\left(\frac{2}{3}\right)^{\frac{1}{3}}\left\{\frac{1}{2} d \tau^{2}+\frac{1}{2} e_{\psi}^{2}+g_{3}^{2}+g_{4}^{2}\right. \\
& \left.+\frac{\tau^{2}}{4}\left(g_{1}^{2}+g_{2}^{2}\right)\right\} .
\end{aligned}
$$

The last term here corresponds to the collapsing sphere $S_{2}$, while the sphere $S_{3}$ associated with three angular terms in the first line has a fixed radius in the limit $\tau \rightarrow 0[12,33]$. The radial coordinate $r$ approaches its minimal value with

$$
\left.r^{3}\right|_{\min }=\left(\frac{3}{2}\right)^{\frac{3}{2}}|b|
$$

at $\tau=0$.

The square root of the determinant of the metric,

$$
\left.\sqrt{g_{6}} \sim|b|^{2} \cosh ^{2} \frac{\tau}{2} \sinh ^{2} \frac{\tau}{2}\right|_{\tau \rightarrow 0} \sim|b|^{2} \tau^{2},
$$

vanishes at $\tau=0$, which shows the degeneration of the conifold metric.

\section{B. NS 3-form at small $\tau$}

In this section we consider an extrapolation of our solution for $\mathrm{H}_{3}$ obtained for large $r$ to the region of small values of $\tau$. For simplicity, we will consider an extrapolation of the solution (3.10), taking $\mu_{2}=0$ in (3.16).

At large values of the radial coordinate $\tau$, the 2-form potential $B_{2}$ is proportional to

$$
B_{2} \sim e_{\theta_{1}} \wedge e_{\varphi_{1}}-e_{\theta_{2}} \wedge e_{\varphi_{2}}
$$

[see (3.3) and (3.9)]. The closed 2-form on the right-hand side can be rewritten in terms of angular differentials $g$. Explicitly, we have

$$
e_{\theta_{1}} \wedge e_{\varphi_{1}}-e_{\theta_{2}} \wedge e_{\varphi_{2}}=g_{1} \wedge g_{2}+g_{3} \wedge g_{4} .
$$

Therefore, it is natural to look for a solution for $B_{2}$ at arbitrary $\tau$ using the ansatz

$$
B_{2}=p(\tau) g_{1} \wedge g_{2}+k(\tau) g_{3} \wedge g_{4},
$$

where $p$ and $k$ are functions of the radial coordinate $\tau$. This ansatz was used in [33] for the type IIB "compactification" on the deformed conifold.

The ansatz (4.11) gives for the field strength

$$
\begin{aligned}
H_{3}= & p^{\prime} d \tau \wedge g_{1} \wedge g_{2}+k^{\prime} d \tau \wedge g_{3} \wedge g_{4} \\
& -\frac{1}{2}(p-k) e_{\psi} \wedge\left(g_{1} \wedge g_{3}+g_{2} \wedge g_{4}\right),
\end{aligned}
$$

where we used the identity [33] 


$$
d\left(g_{1} \wedge g_{2}-g_{3} \wedge g_{4}\right)=-e_{\psi} \wedge\left(g_{1} \wedge g_{3}+g_{2} \wedge g_{4}\right) .
$$

Here, primes denote derivatives with respect to $\tau$.

In particular, at large $\tau$ we have $p=k=\mu_{1} / 3$, where we rewrite solution (3.10) in terms of $\tau$ using relation (4.5) at $\tau \rightarrow \infty$.

Calculating the 10D-dual of (4.12), we get

$$
\begin{aligned}
* H_{3}= & T^{2} d x^{0} \wedge d x^{1} \wedge d x^{2} \wedge d x^{3} \\
& \wedge\left\{p^{\prime} \operatorname{coth}^{2}\left(\frac{\tau}{2}\right) e_{\psi} \wedge g_{3} \wedge g_{4}\right. \\
& +k^{\prime} \tanh ^{2}\left(\frac{\tau}{2}\right) e_{\psi} \wedge g_{1} \wedge g_{2} \\
& \left.-\frac{1}{2}(p-k) d \tau \wedge\left(g_{1} \wedge g_{3}+g_{2} \wedge g_{4}\right)\right\} .
\end{aligned}
$$

Then the equation of motion (3.5) gives two equations:

$$
\begin{aligned}
4 \partial_{\tau}\left(\frac{p^{\prime}}{\tau^{2}}\right)-\frac{1}{2}(p-k) & =0, \\
\frac{1}{2} \partial_{\tau}\left(\tau^{2} k^{\prime}\right)+(p-k) & =0,
\end{aligned}
$$

which we write down in the limit of small $\tau$. To derive (4.15) we used the identity [33]

$$
d\left(g_{1} \wedge g_{3}+g_{2} \wedge g_{4}\right)=e_{\psi} \wedge\left(g_{1} \wedge g_{2}-g_{3} \wedge g_{4}\right) .
$$

The solution of these equations at small $\tau$ has the form

$$
k(\tau) \approx \mu_{1} \tau, \quad p(\tau) \approx-\frac{\mu_{1}}{80} \tau^{5},
$$

up to an overall constant.

To conclude this section, we note that at $\tau=0$ our solution (4.12) tends to a constant,

$$
H_{3}(\tau=0)=\mu_{1} d \tau \wedge g_{3} \wedge g_{4},
$$

which we impose as boundary conditions at $S_{3}$, which does not shrink at $\tau=0$. These boundary conditions ensure a nonzero solution for $\mathrm{H}_{3}$.

\section{The potential for the baryon $b$ at large $|\boldsymbol{b}|$}

Substituting solutions for $\mathrm{H}_{3}$ and its 10D-dual given by (4.12) and (4.14) to the kinetic term (3.24), we get

$$
-\frac{e^{-\Phi}}{12} \int H_{3} \wedge * H_{3} \sim-\mu_{1}^{2} T^{2} e^{-\Phi} \int d^{4} x d \tau \tau^{2},
$$

where only the function $k(\tau)$ (4.17) contributes to the leading order in $\tau$. Thus, the potential for $b$ takes the form

$$
V(b)=\text { const } \mu_{1}^{2} T^{2} e^{-\Phi} \tau_{\max }^{3},
$$

where $\tau_{\max }$ is the infrared cutoff with respect to the radial coordinate $\tau$, related to $R_{\mathrm{IR}}$ as follows:

$$
|b| \cosh \left(\tau_{\max }\right)=\left(\frac{2}{3}\right)^{\frac{3}{2}} R_{\mathrm{IR}}^{3}
$$

[see (4.5)].

As we already explained, we expect that in our runaway vacuum, $b$ is large and close to $R_{\mathrm{IR}}$, and therefore $\tau_{\max }$ is small. Expanding $\cosh \tau$ at small $\tau$, we get

$$
\tau_{\max } \sim \sqrt{\frac{R_{\mathrm{IR}}^{3}-\left(\frac{3}{2}\right)^{\frac{3}{2}}|b|}{|b|}} .
$$

This gives the potential for the baryon $b$ at large $|b|$ :

$$
V(b)=\text { const } \mu_{1}^{2} T^{2} e^{-\Phi}\left[\frac{R_{\mathrm{IR}}^{3}-\left(\frac{3}{2}\right)^{\frac{3}{2}}|b|}{|b|}\right]^{\frac{3}{2}} .
$$

We see that to minimize the potential, $|b|$ becomes large and approaches the infrared cutoff

$$
\langle|b|\rangle=\left(\frac{2}{3}\right)^{\frac{3}{2}} R_{\mathrm{IR}}^{3} \rightarrow \infty .
$$

As we expected earlier in Sec. III D, we get a runaway vacuum.

In fact, $\tau_{\max }^{3}$, which enters (4.20), is the volume of the three-dimensional ball bounded by the sphere $S_{2}$ of the conifold with the maximum radius $\tau_{\max }$. It shrinks to zero as $b$ tends to its VEV (4.24). To avoid singularities we can regularize the size of $S_{2}$ by introducing small nonzero $\beta$, which makes the conifold "slightly resolved" [see (2.8)]. We take the limit $\beta \rightarrow 0$ at the last step. Then the value of the potential and all its derivatives vanish in the vacuum (4.24) at $|b|=\langle|b|\rangle$; for example,

$$
\left.V(b)\right|_{|b|=\langle|b|\rangle}=\text { const } \mu_{1}^{2} T^{2} e^{-\Phi} \frac{\beta^{3}}{R_{\mathrm{IR}}^{9 / 2}} \rightarrow 0 .
$$

In particular, the mass term for $b$ is zero. This is in accordance with $\mathcal{N}=2$ supersymmetry in 4D SQCD, since the mass of $\tilde{b}$ is zero [see (3.23)].

To summarize, the $\mathrm{H}_{3}$-form flux produces the following effects.

(i) The Higgs branch of the baryon $b$ in $4 \mathrm{D}$ SQCD is lifted.

(ii) The vacuum is of a runaway type, $\langle|b|\rangle \rightarrow \infty$.

(iii) At the runaway vacuum the sphere $S_{2}$ of the conifold degenerates, while the radius of the sphere $S_{3}$ tends to infinity. 
We will interpret this degeneration in terms of $\mathcal{N}=2$ SQCD in Sec. VB.

\section{INTERPRETATION IN TERMS OF 4D SQCD}

\section{A. 3-form flux in terms of quark masses}

In this section we interpret the NS 3-form $\mathrm{H}_{3}$ in terms of quark masses of $4 \mathrm{D} \mathcal{N}=2 \mathrm{SQCD}$. For $N_{f}=4$ we have four complex mass parameters. However, a shift of the complex scalar $a$, a superpartner of the U(1) gauge field, produces an overall shift of quark masses. This can be also seen in the $\mathbb{W C P}(2,2)$ model $(2.3)$ on the world sheet of the non-Abelian string; a constant shift of the scalar $\sigma$ makes an overall shift of quark masses. Thus, in fact, we have three independent complex mass parameters in our 4D SQCD. For example, we can choose three mass differences,

$$
m_{1}-m_{2}, \quad m_{3}-m_{4}, \quad m_{1}-m_{3},
$$

as independent parameters.

On the string theory side, our solution (3.16) for the 3 -form $H_{3}$ is parametrized by two real parameters, $\mu_{1}$ and $\mu_{2}$. Thus, we expect that the nonzero $\mathrm{H}_{3}$-flux can be interpreted in terms of a particular choice of quark masses, subject to two complex constraints.

One constraint we already discussed. We know that $H_{3}$ does not produce a mass term for the $b$-baryon. This ensures that

$$
m_{1}+m_{2}-m_{3}-m_{4}=0
$$

[see (1.2)].

To derive the second constraint, we consider the exact twisted superpotential for the $\operatorname{WCP}(2,2)$ model (2.3) obtained by integrating out $n$ and $\rho$ supermultiplets. It is a generalization $[37,38]$ of the $\mathbb{C P}(N-1)$ model superpotential $[30,39-41]$ of the Veneziano-Yankielowicz type [42]. In the present case $N_{f}=2 N=4$, it reads:

$$
\begin{aligned}
\mathcal{W}_{\mathrm{WCP}}(\sigma)= & \frac{1}{4 \pi}\left\{\sum_{P=1,2}\left(\sqrt{2} \sigma+m_{P}\right) \ln \left(\sqrt{2} \sigma+m_{P}\right)\right. \\
& -\sum_{K=3,4}\left(\sqrt{2} \sigma+m_{K}\right) \ln \left(\sqrt{2} \sigma+m_{K}\right) \\
& +2 \pi \sqrt{2} \sigma \beta+\text { const }\},
\end{aligned}
$$

where we use one and the same notation $\sigma$ for the twisted superfield [30] and its lowest scalar component.

To study the vacuum structure of the theory, we minimize this superpotential with respect to $\sigma$ to obtain the $2 \mathrm{D}$ vacuum equation

$$
\prod_{P=1,2}\left(\sqrt{2} \sigma+m_{P}\right)=e^{-2 \pi \beta} \prod_{K=3,4}\left(\sqrt{2} \sigma+m_{K}\right) .
$$

Consider the limit $\beta \rightarrow 0$ in this equation. In this limit, (5.4) reduces to the following quadratic equation:

$$
\begin{aligned}
& 2 \pi \beta(\sqrt{2} \sigma)^{2}+(\sqrt{2} \sigma)\left(m_{1}+m_{2}-m_{3}-m_{4}\right) \\
& \quad+m_{1} m_{2}-m_{3} m_{4}=0 .
\end{aligned}
$$

The second term here is zero due to the constraint (5.2). Two roots of (5.5) read

$$
\sqrt{2} \sigma= \pm\left.\left[\frac{1}{2 \pi \beta}\left(m_{1} m_{2}-m_{3} m_{4}\right)\right]^{1 / 2}\right|_{\beta \rightarrow 0} \rightarrow \infty .
$$

However, infinite VEVs of $\sigma$ cost infinite energy for nonzero $\mathrm{H}_{3}$. To see this, observe that from the string theory side we learned that the VEV of $b$ goes to infinity [see (4.24)]. Then Eq. (4.5) shows that the radial coordinate $\tilde{r}$ is very large, which means that (some of) the $n$ 's and $\rho$ 's become infinitely large [see (2.11)]. Given the infinite VEV of $\sigma$ this makes first two terms in the second line of the $\mathbb{W} C \mathbb{P}(2,2)$ action $(2.3)$ infinite.

To avoid this we impose the second constraint, namely

$$
m_{1} m_{2}-m_{3} m_{4}=0 \text {. }
$$

Now, let us solve two constraints in (5.2) and (5.7) for masses $m_{3}$ and $m_{4}$ as functions of $m_{1}$ and $m_{2}$. Finding, say, $m_{4}$ from (5.2) and substituting it into (5.7), we get a quadratic equation for $m_{3}$,

$$
m_{3}^{2}-m_{3}\left(m_{1}+m_{2}\right)+m_{1} m_{2}=0 .
$$

Two roots of this equation read

$$
m_{3}=m_{1}, \quad m_{4}=m_{2}
$$

and

$$
m_{3}=m_{2}, \quad m_{4}=m_{1} .
$$

These two options are essentially the same, up to the permutation of quarks $q^{3}$ and $q^{4}$. Let us choose the first option in (5.9).

The above arguments lead us to the conclusion that the $\mathrm{H}_{3}$-flux can be interpreted in terms of the single mass difference $\left(m_{1}-m_{2}\right)$. Thus, we complexify the parameters $\mu_{1,2}$ and identify

$$
\mu \equiv \mu_{1}+i \mu_{2}=\sqrt{\frac{g_{s}}{T}}\left(m_{1}-m_{2}\right), \quad m_{3}=m_{1}, \quad m_{4}=m_{2} .
$$

Now, in terms of quark masses our solution (3.16) for the NS 3-form reads 
$H_{3}=\sqrt{\frac{g_{s}}{T}}\left[\operatorname{Re}\left(m_{1}-m_{2}\right) \alpha_{3}+\frac{1}{3} \operatorname{Im}\left(m_{1}-m_{2}\right) \beta_{3}\right]$,

while the potential (3.26) for the baryon $b$ becomes

$$
V(b)=\operatorname{const} T\left|m_{1}-m_{2}\right|^{2} \log \frac{R_{\mathrm{IR}}^{3}}{|b|} .
$$

Similarly we can rewrite our solution (4.12) for $\mathrm{H}_{3}$ obtained at large $|b|$ in terms of $\left(m_{1}-m_{2}\right)$.

\section{B. Degeneration of the conifold}

If we take the limit of large $\left|m_{1}-m_{2}\right| \gg \sqrt{\xi}$ in 4D SQCD keeping the constraint (5.9), non-Abelian degrees of freedom decouple and $\mathrm{U}(2)$ gauge theory flows to $\mathcal{N}=2$ supersymmetric Abelian theory with the gauge group $\mathrm{U}(1) \times \mathrm{U}(1)$ and $N_{f}=4$ quark flavors. For example, offdiagonal gauge fields acquire large masses $\sim\left|m_{1}-m_{2}\right|$ and decouple. ${ }^{8}$ However, in this paper we consider the limit of small quark masses on the string theory side and therefore cannot make $\left|m_{1}-m_{2}\right|$ large. At small $\left|m_{1}-m_{2}\right| \ll \sqrt{\xi}$, our 4D SQCD remains to be $\mathrm{U}(2)$ gauge theory.

On the other hand, in the world sheet $\mathbb{W} \mathbb{C P}(2,2)$ model on the non-Abelian string the story is different. This theory is conformal and has no scale. Therefore, once we switch on nonzero $\left(m_{1}-m_{2}\right)$ there is no notion of smallness of this deformation. We see a dramatic effect as soon as $\left(m_{1}-m_{2}\right)$ becomes nonzero.

Classically there are two branches in the $\operatorname{WCP}(2,2)$ model (2.3) associated with VEVs of $\sigma$,

$$
\sqrt{2} \sigma=-m_{1}
$$

or

$$
\sqrt{2} \sigma=-m_{2}
$$

Let us choose the first branch for definiteness. Then fields $n^{P=2}$ and $\rho^{K=4}$ acquire masses $\left|m_{1}-m_{2}\right|$ and decouple in the infrared. The low energy effective theory at scales below $\left|m_{1}-m_{2}\right|$ is the $\mathbb{W C P}(1,1)$ model,

$$
\begin{aligned}
S= & \int d^{2} x\left\{\left|\nabla_{\alpha} n^{1}\right|^{2}+\left|\tilde{\nabla}_{\alpha} \rho^{3}\right|^{2}+\frac{1}{4 e^{2}} F_{\alpha \beta}^{2}\right. \\
& +\frac{1}{e^{2}}\left|\partial_{\alpha} \sigma\right|^{2}+2|\sigma|^{2}\left|n^{1}\right|^{2}+2|\sigma|^{2}\left|\rho^{3}\right|^{2} \\
& \left.+\frac{e^{2}}{2}\left(\left|n^{1}\right|^{2}-\left|\rho^{3}\right|^{2}-\operatorname{Re} \beta\right)^{2}\right\},
\end{aligned}
$$

\footnotetext{
${ }^{8}$ In addition to masses $m_{G} \sim g \sqrt{\xi}$ due to the Higgs mechanism (see [9] for a review).
}

where much in the same way as in (2.3), the limit $e^{2} \rightarrow \infty$ is assumed and we made the shift $\left(\sqrt{2} \sigma+m_{1}\right) \rightarrow \sqrt{2} \sigma$.

The number of real degrees of freedom in (5.16) is $4-1-1=2$, where 4 is the number of real degrees of freedom of $n^{1}$ and $\rho^{3}$ and, much in the same way as in (2.3), we subtract 2 due to the $D$-term constraint in (5.16) and the $\mathrm{U}(1)$ phase eaten by the Higgs mechanism.

Physically, the $\mathbb{W C P}(1,1)$ model describes an Abelian semilocal vortex string supported in the $\mathcal{N}=2$ supersymmetric U(1) gauge theory with $N_{f}=2$ quark flavors. This vortex has no orientational moduli, but it has one complex size modulus $\rho^{3}$ (see [24-26]). Thus, we can think that upon switching on $\left(m_{1}-m_{2}\right)$ a non-Abelian string flows to an Abelian one.

The low-energy $\mathbb{W C P}(1,1)$ model is also conformal. Moreover, it was shown in [43] that in the nonlinear sigma model formulation it flows to a free theory on $\mathbb{R}^{2}$ in the infrared. Thus, in fact, switching on $\left(m_{1}-m_{2}\right)$ with the constraint (5.9) does not break the conformal invariance on the world sheet; it just reduces the number of degrees of freedom transforming a non-Abelian string into an Abelian one. The string theory which one would associate with the sigma model (5.16) is noncritical.

This supports our interpretation of the flux "compactification" on the conifold in terms of quark masses. On the string theory side, switching on $\left(m_{1}-m_{2}\right)$ is reflected in the degeneration of the conifold, which effectively reduces its dimension. Also in the limit $|b| \rightarrow \infty$, the radius of the sphere $S_{3}$ of the conifold becomes infinite and it tends to a flat three-dimensional space. This matches the field theory result [43] that the $\mathbb{W} C P(1,1)$ model flows to a free theory in the infrared. It would be tempting to interpret the extra coordinate of the sphere $S_{3}$ of the conifold in the limit $|b| \rightarrow \infty$ as a Liouville coordinate for a noncritical string associated with the sigma model (5.16). This is left for a future work.

To conclude, we note that although 4D SQCD gets just slightly deformed as we switch on small $\left(m_{1}-m_{2}\right)$ at weak coupling, this deformation becomes much more pronounced at strong coupling. Namely, at $\tau_{S W}=1$ [which corresponds to $\beta=0$ in (2.3)] the nonperturbative Higgs branch gets lifted and we have a runaway vacuum (4.24) for the stringy baryon $b$.

\section{CONCLUSIONS}

In this paper we considered the NS 3-form flux "compactification" for the string theory of the critical nonAbelian vortex supported in $\mathcal{N}=2$ SQCD with gauge group $\mathrm{U}(2)$ and $N_{f}=4$ quark flavors. Using the supergravity approach, we found a solution for the 3 -form $\mathrm{H}_{3}$ to the leading order at small $\mathrm{H}_{3}$ neglecting the back reaction on the conifold metric and the dilaton. The nonzero 3-form $\mathrm{H}_{3}$ generates a potential for the complex structure modulus $b$ of the conifold, which is interpreted as a BPS baryonic 
hypermultiplet in 4D SQCD at strong coupling. This potential lifts the Higgs branch formed by VEVs of $b$ and leads to a runaway for $b,\langle|b|\rangle \rightarrow \infty$.

We interpret the 3-form $\mathrm{H}_{3}$ as a quark mass deformation of $4 \mathrm{D} \mathrm{SQCD}$. The reason for this interpretation is that there is no other deformation in $4 \mathrm{D}$ SQCD preserving $\mathcal{N}=2$ supersymmetry, which produces a scalar potential. We use field theory arguments to relate the 3-form $\mathrm{H}_{3}$ to the quark mass difference $\left(m_{1}-m_{2}\right)$ [see (5.12)] subject to the constraint (5.9).

At the runaway vacuum the conifold degenerates to lower dimensions. This qualitatively matches with a flow to the $\mathbb{W C P}(1,1)$ model $(5.16)$ on the string world sheet, which is expected if one switches on the mass difference $\left(m_{1}-m_{2}\right)$. This flow can be thought of as a flow from a non-Abelian string to an Abelian one.

As a direction of a future research we can mention the finding of exact solutions of 10D supergravity equations with a nonzero NS 3-form flux. This would allow us to describe the limit of large $\left(m_{1}-m_{2}\right)$ in terms of the string theory. In this limit, non-Abelian degrees of freedom in 4D SQCD with $U(2)$ gauge group decouple and the theory flows to an Abelian $\mathrm{U}(1) \times \mathrm{U}(1)$ theory.

Another challenging problem is to find supergravity deformations associated with other choices of quark masses in 4D SQCD.

\section{ACKNOWLEDGMENTS}

The author is grateful to A. Gorsky, E. Ievlev, A. Losev, and M. Shifman for very useful and stimulating discussions. This work was supported by William I. Fine Theoretical Physics Institute of the University of Minnesota.

\section{APPENDIX: KINETIC TERM FOR $\tilde{B}$-BARYON}

In this Appendix we calculate kinetic terms for $4 \mathrm{D}$ scalars $\tilde{b}_{1,2}$ which arise from the expansion of the $10 \mathrm{D}$ 3 -form potential $C_{3}$ in (3.19). Assuming that scalars $\tilde{b}_{1,2}$ depend only on $4 \mathrm{D}$ coordinates $x_{\mu}$ and dropping the $4 \mathrm{D}$ 3-form $C_{3}^{4 D}$ (we consider it in Sec. III C), we write

$$
F_{4}=d C_{3}=\partial_{\mu} \tilde{b}_{1} d x^{\mu} \wedge \alpha_{3}+\frac{1}{3} \partial_{\mu} \tilde{b}_{2} d x^{\mu} \wedge \beta_{3} .
$$

Calculating the 10D-dual of this form we get

$$
\begin{aligned}
* F_{4}= & -\frac{1}{33 !} T^{2} \varepsilon_{\mu \nu \gamma \delta} \partial^{\mu} \tilde{b}_{1} d x^{\nu} \wedge d x^{\gamma} \wedge d x^{\delta} \wedge \beta_{3} \\
& +\frac{1}{3 !} T^{2} \varepsilon_{\mu \nu \gamma \delta} \partial^{\mu} \tilde{b}_{2} d x^{\nu} \wedge d x^{\gamma} \wedge d x^{\delta} \wedge \alpha_{3},
\end{aligned}
$$

where we used (3.14).

Now, writing the kinetic term for $C_{3}$ in (3.1) as

$$
\int F_{4} \wedge * F_{4}
$$

we finally get

$$
S_{\text {kin }}(\tilde{b})=T \int d^{4} x\left\{\left|\partial_{\mu} \tilde{b}_{1}\right|^{2}+\left|\partial_{\mu} \tilde{b}_{2}\right|^{2}\right\} \log \frac{R_{\mathrm{IR}}^{3}}{|b|},
$$

where we used (3.17) and (3.18).

We see that scalars $\tilde{b}_{1,2}$ have the same logarithmic metric as the complex scalar $b$. As we already mentioned, the complexified scalar $\tilde{b}$, together with $b$, forms a bosonic part of the 4D baryonic hypermultiplet.
[1] M. Shifman and A. Yung, Critical string from non-Abelian vortex in four dimensions, Phys. Lett. B 750, 416 (2015).

[2] A. Hanany and D. Tong, Vortices, instantons and branes, J. High Energy Phys. 07 (2003) 037.

[3] R. Auzzi, S. Bolognesi, J. Evslin, K. Konishi, and A. Yung, Non-Abelian superconductors: Vortices and confinement in $\mathcal{N}=2$ SQCD, Nucl. Phys. B673, 187 (2003).

[4] M. Shifman and A. Yung, Non-Abelian string junctions as confined monopoles, Phys. Rev. D 70, 045004 (2004).

[5] A. Hanany and D. Tong, Vortex strings and fourdimensional gauge dynamics, J. High Energy Phys. 04 (2004) 066.

[6] A. Abrikosov, On the magnetic properties of superconductors of the second group, Sov. Phys. JETP 32, 1442 (1957); H. Nielsen and P. Olesen, Vortex-line models for dual strings, Nucl. Phys. B61, 45 (1973); Reprinted in Solitons and Particles, edited by C. Rebbi and G. Soliani (World Scientific, Singapore, 1984), p. 365.

[7] D. Tong, TASI lectures on solitons, arXiv:hep-th/0509216.

[8] M. Eto, Y. Isozumi, M. Nitta, K. Ohashi, and N. Sakai, Solitons in the Higgs phase: The moduli matrix approach, J. Phys. A 39, R315 (2006).

[9] M. Shifman and A. Yung, Supersymmetric solitons and how they help us understand non-Abelian gauge theories, Rev. Mod. Phys. 79, 1139 (2007); For an expanded version see Supersymmetric Solitons, (Cambridge University Press, Cambridge, England, 2009).

[10] D. Tong, Quantum vortex strings: A review, Ann. Phys. (Amsterdam) 324, 30 (2009).

[11] P. Koroteev, M. Shifman, and A. Yung, Non-Abelian vortex in four dimensions as a critical string on a conifold, Phys. Rev. D 94, 065002 (2016). 
[12] P. Candelas and X. C. de la Ossa, Comments on conifolds, Nucl. Phys. B342, 246 (1990).

[13] A. Neitzke and C. Vafa, Topological strings and their physical applications, arXiv:hep-th/0410178.

[14] P. Koroteev, M. Shifman, and A. Yung, Studying critical string emerging from non-Abelian vortex in four dimensions, Phys. Lett. B 759, 154 (2016).

[15] M. Shifman and A. Yung, Critical non-Abelian vortex in four dimensions and little string theory, Phys. Rev. D 96, 046009 (2017).

[16] M. Shifman and A. Yung, Hadrons of $\mathcal{N}=2$ supersymmetric QCD in four dimensions from little string theory, Phys. Rev. D 98, 085013 (2018).

[17] D. Kutasov, Introduction to little string theory, in Superstrings and Related Matters 2001, edited by C. Bachas, K. S. Narain, and S. Randjbar-Daemi (Proceedings of the ICTP Spring School of Physics, Triest, 2002), pp. 165-209.

[18] J. Louis, Generalized Calabi-Yau compactifications with D-branes and fluxes, Fortschr. Phys. 53, 770 (2005).

[19] E. Ievlev, M. Shifman, and A. Yung, String baryon in fourdimensional $\mathrm{N}=2$ supersymmetric QCD from the 2D-4D correspondence, Phys. Rev. D 102, 054026 (2020).

[20] J. Louis and A. Micu, Type II theories compactified on Calabi-Yau threefolds in the presence of background fluxes, Nucl. Phys. B635, 395 (2002).

[21] S. Kachru and A. Kashani-Poor, Moduli potentials in type IIA compactifications with RR and NS flux, J. High Energy Phys. 03 (2005) 066.

[22] P. Fayet and J. Iliopoulos, Spontaneously broken supergauge symmetries and goldstone spinors, Phys. Lett. 51B, 461 (1974).

[23] D. Tong, Monopoles in the Higgs phase, Phys. Rev. D 69, 065003 (2004).

[24] For a review see e.g., A. Achucarro and T. Vachaspati, Semilocal and electroweak strings, Phys. Rep. 327, 347 (2000).

[25] M. Shifman and A. Yung, Non-Abelian semilocal strings in $\mathcal{N}=2$ supersymmetric QCD, Phys. Rev. D 73, 125012 (2006).

[26] M. Eto, J. Evslin, K. Konishi, G. Marmorini, M. Nitta, K. Ohashi, W. Vinci, and N. Yokoi, On the moduli space of semilocal strings and lumps, Phys. Rev. D 76, 105002 (2007).

[27] M. Shifman, W. Vinci, and A. Yung, Effective world-sheet theory for non-Abelian semilocal strings in $\mathcal{N}=2$ supersymmetric QCD, Phys. Rev. D 83, 125017 (2011).
[28] P. Koroteev, M. Shifman, W. Vinci, and A. Yung, Quantum dynamics of low-energy theory on semilocal non-Abelian strings, Phys. Rev. D 84, 065018 (2011).

[29] J. Chen, C. H. Sheu, M. Shifman, G. Tallarita, and A. Yung, Long way to Ricci flatness, J. High Energy Phys. 10 (2020) 059 .

[30] E. Witten, Phases of $\mathrm{N}=2$ theories in two dimensions, Nucl. Phys. B403, 159 (1993).

[31] J. Polchinski and A. Strominger, Effective String Theory, Phys. Rev. Lett. 67, 1681 (1991).

[32] K. Ohta and T. Yokono, Deformation of conifold and intersecting branes, J. High Energy Phys. 02 (2000) 023.

[33] I. R. Klebanov and M. J. Strassler, Supergravity and a confining gauge theory: Duality cascades and chiSBresolution of naked singularities, J. High Energy Phys. 08 (2000) 052.

[34] I. R. Klebanov and N. A. Nekrasov, Gravity duals of fractional branes and logarithmic RG flow, Nucl. Phys. B574, 263 (2000).

[35] I. R. Klebanov and A. A. Tseytlin, Gravity duals of supersymmetric $\mathrm{SU}(\mathrm{N}) \mathrm{SU}(\mathrm{N}+\mathrm{M})$ gauge theories, Nucl. Phys. B578, 123 (2000).

[36] L. A. P. Zayas and A. A. Tseytlin, 3-branes on resolved conifold, Phys. Rev. D 63, 086006 (2001).

[37] A. Hanany and K. Hori Branes and $\mathrm{N}=2$ theories in two dimensions, Nucl. Phys. B513, 119 (1998).

[38] N. Dorey, T. J. Hollowood, and D. Tong, The BPS spectra of gauge theories in two and four dimensions, J. High Energy Phys. 05 (1999) 006.

[39] A. D'Adda, A. C. Davis, P. DiVeccia, and P. Salamonson, An effective action for the supersymmetric $\mathrm{CP}^{n-1}$ models, Nucl. Phys. B222, 45 (1983).

[40] S. Cecotti and C. Vafa, On classification of $\mathcal{N}=2$ supersymmetric theories, Commun. Math. Phys. 158, 569 (1993).

[41] N. Dorey, The BPS spectra of two-dimensional supersymmetric gauge theories with twisted mass terms, J. High Energy Phys. 11 (1998) 005.

[42] G. Veneziano and S. Yankielowicz, An effective lagrangian for the pure $\mathrm{N}=1$ supersymmetric Yang-Mills theory, Phys. Lett. 113B, 231 (1982).

[43] O. Aharony, S. S. Razamat, N. Seiberg, and B. Willett, The long flow to freedom, J. High Energy Phys. 02 (2017) 056. 